\title{
To Assess Correlation of Fasting Blood Glucose, Hb1Ac and Serum Lipid Level in Type II Diabetes Mellitus Patients
}

\author{
Nagendra Singh Chauhan', Hemendra Kumar Agrawal ${ }^{2}$ \\ ${ }^{1}$ Associate Professor, Department of Medicine, FH Medical College, Tundla, ${ }^{2}$ Assistant Professor, Department of Medicine, FH Medical College, Tundla.
}

\section{Abstract}

Background: Diabetes Mellitus (DM) refers to a group of common metabolic disorder that share the phenotype of hyperglycaemia caused due to either deficiency of insulin secretion or insulin resistance. The present study was conducted to assess correlation of fasting blood glucose, Hb1Ac and type II DM in given adults. Subjects and Methods: The present study was conducted on 134 patients with type II diabetes mellitus (DM) of both genders. Patients were subjected to estimation of fasting and random blood glucose level. Modified method of Fluckiger and Winterhalter was used for estimation of glycosylated haemoglobin (HbA1C). Results: Out of 134 patients, males were 84 and females were 50. Age group 20-30 years had 11, 30-40 years had 14, 40-50 years had 38, 50-60 years had 46 and >60 years had 25 patients. The mean fasting blood glucose level in patients with good degree of control was 106.2, in fair was 164.4 and in poor was 208.2. Glycosylated hemoglobin level was good (6.74), fair (8.12) and poor (12.54). A positive correlation of fasting blood glucose and glycosylated hemoglobin level and serum cholesterol \& glycosylated hemoglobin level $(\mathrm{P}<0.05)$ was found. Conclusion: Authors found a positive correlation of fasting blood glucose and glycosylated hemoglobin level and serum cholesterol \& glycosylated hemoglobin level in type II diabetes mellitus patients.

Keywords: Diabetes Mellitus, Fasting blood glucose, Glycosylated hemoglobin

Corresponding Author: Dr. Hemendra Kumar Agrawal, Assistant Professor, Department of Medicine, FH Medical College, Tundla.

Received: July 2019

Accepted: August 2019

\section{Introduction}

Diabetes Mellitus (DM) refers to a group of common metabolic disorder that share the phenotype of hyperglycaemia caused due to either deficiency of insulin secretion or insulin resistance. Diabetes Mellitus is one of the most common endocrinal Diseases in the world. Incidences of this disease are increasing worldwide and this disease is called disease of this millennium. ${ }^{[1]}$ The worldwide prevalence of this disease has risen dramatically over the past two decades, from an estimated 30 million in 1985 to 85 million in 2010 and 415 million in 2017. India is thought to be capital for DM, the incidences of this disease is 73 million in 2015. Diabetes mellitus is commonly associated with abnormalities of carbohydrate metabolism, lipid metabolism, insulin resistance etc. ${ }^{[2]}$

In order to detect diabetics, fasting blood glucose (FBS) is suggested as the best and the most common test with the cutoff point $>126 \mathrm{mg} / \mathrm{dl} .{ }^{[3]}$ However, there are some issues about using FBS such as keeping the clients fast for about 8 hours and not being applicable in the afternoon. Besides, in centralized screening when laboratory facilities are available, HbA1c test, which is the percentage of glycatedhemoglobin is recommended to measure the incidence or prevalence. Apart from the efficacy of HbAlc in detection of diabetes, it is an important marker to assess the microvascular complications and plasma glucose. ${ }^{[4]}$ The relationship between HbAlc and blood glucose is documented in the literature denoting a straight relationship. However, this relationship has not been confirmed by others. There is a controversy about the performance of HbA1c in case finding. It has been argued that due to problems in standardization and variations in styles of $\mathrm{HbA} 1 \mathrm{c}$ test, it is not recommended as a routine test for screening of diabetes. ${ }^{[5]}$ The present study was conducted to assess correlation of fasting blood glucose, Hb1 Ac and type II DM in given adults.

\section{Subjects and Methods}

The present study was conducted in the department of General Medicine. It comprised of 134 patients with type II diabetes mellitus (DM) of both genders. The study was approved from institutional ethical committee. All participants were informed regarding the study and written consent was obtained.

Data such as name, age, gender etc. was recorded. Patients were subjected to estimation of fasting and random blood glucose level. Modified method of Fluckiger and Winterhalter was used for estimation of glycosylated haemoglobin (HbA1C). The plasma lipoproteins were estimated by electrophoretic separation of lipoproteins on agarose gel. Plasma cholesterol was determined by libermanBuchard reaction. Results thus obtained were subjected to 
statistical analysis. $\mathrm{P}$ value less than 0.05 was considered significant.

\section{Results}

Table 1: Distribution of patients

\begin{tabular}{|l|l|l|}
\hline Total- 134 & Males & Females \\
\hline Gender & 84 & 50 \\
\hline Number &
\end{tabular}

[Table 1] shows that out of 134 patients, males were 84 and females were 50 .

\begin{tabular}{|c|c|c|}
\hline Age group (Years) & Number & P value \\
\hline $20-30$ & 11 & \multirow[t]{5}{*}{0.02} \\
\hline $30-40$ & 14 & \\
\hline $40-50$ & 38 & \\
\hline $50-60$ & 46 & \\
\hline$>60$ & 25 & \\
\hline
\end{tabular}

[Table 2, Figure1] shows that age group 20-30 years had 11, 30-40 years had 14, 40-50 years had 38, 50-60 years had 46 and $>60$ years had 25 patients. The difference was significant $(\mathrm{P}<0.05)$.

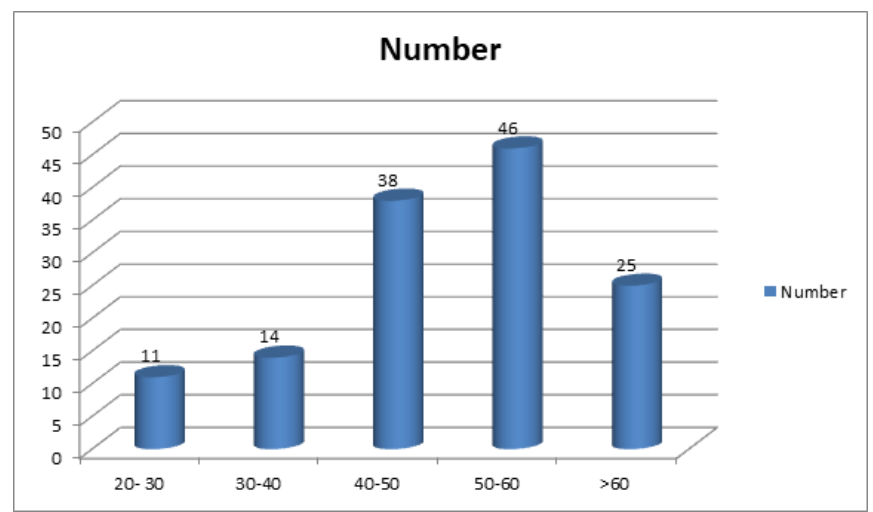

Figure 1: Age wise distribution of patients

Table 3: Assessment of blood glucose level
\begin{tabular}{|l|l|l|l|}
\hline Blood glucose & Good & Fair & Poor \\
\hline FBG $(\mathrm{mg} / \mathrm{dl})$ & 106.2 & 164.4 & 208.2 \\
\hline HbA1C $(\%)$ & 6.74 & 8.12 & 12.54 \\
\hline
\end{tabular}

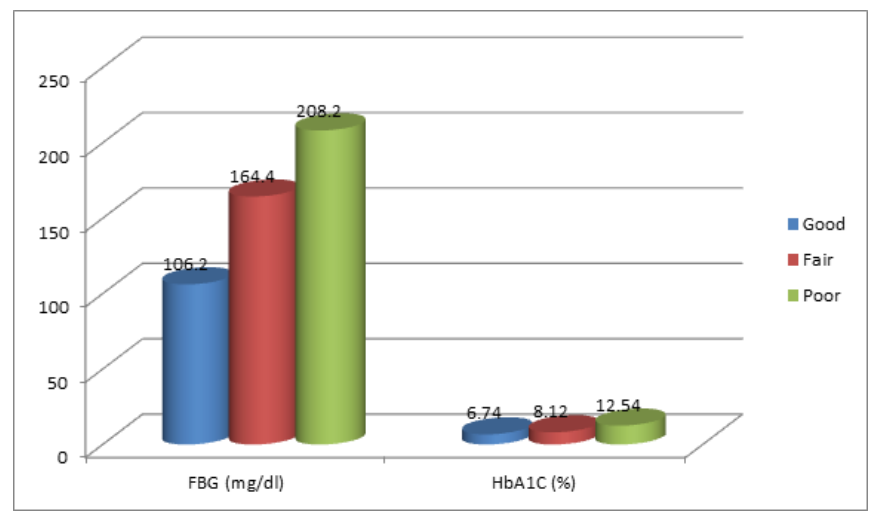

Figure 2: Assessment of blood glucose level

[Table 3, Figure 2] shows that mean fasting blood glucose level in patients with good degree of control was 106.2, in fair was 164.4 and in poor was 208.2. Glycosylated hemoglobin level was good (6.74), fair (8.12) and poor (12.54).

Table 4: Correlation of fasting blood glucose and glycosylated hemoglobin level and serum cholesterol

\begin{tabular}{|l|l|l|}
\hline Parameters & Correlation & Significance \\
\hline FBG \& HbA1C & 0.392 & 0.03 \\
\hline HbA1C \& cholesterol & 0.387 & 0.04 \\
\hline
\end{tabular}

[Table 4] shows a positive correlation of fasting blood glucose and glycosylated hemoglobin level and serum cholesterol \& glycosylated hemoglobin level $(\mathrm{P}<0.05)$ was found.

\section{Discussion}

Type 2 diabetes mellitus (DM) is a chronic metabolic disorder in which prevalence has been increasing steadily all over the world. As a result of this trend, it is fast becoming an epidemic in some countries of the world with the number of people affected expected to double in the next decade due to increase in ageing population, thereby adding to the already existing burden for healthcare providers, especially in poorly developed countries.

The United Kingdom Prospective Diabetes Study considered 6.2 as the normal level, while many laboratories consider 4-6 as a normal range. It seems that in different settings such as screening, diagnosis and prediction of progression of diabetes we need to define different cut off points. ${ }^{[6]}$ For example, It is suggested the value of $6.5 \%$ or greater as a diabetes diagnostic criterion and $6 \%$ and 4.7 for screening test. Inoue and his colleagues used the value of $5.8 \%$ for the prediction of progression of diabetes type 2 , and the value $<7$ as a good predictive of satisfactory blood glucose control in type 1 diabetes. The main aim of major primary studies carried out in diabetes in Iran was to recognize the range of HbA1c in the diabetics, tracing back the complications of diabetes and diabetes control. ${ }^{[7]} \mathrm{A}$ few researches have been carried out to find out the cutoff value of $\mathrm{HbAlc}$ in screening; however, they mainly used a selective samples mainly focusing on high risk groups. Furthermore, a study has determined the normal range of HbA1c in a sample of non-diabetics. ${ }^{[8]}$ The present study was conducted to assess correlation of fasting blood glucose, Hb1Ac and type II DM in given adults.

In present study, out of 134 patients, males were 84 and females were 50. We found that age group 20-30 years had $11,30-40$ years had $14,40-50$ years had $38,50-60$ years had 46 and $>60$ years had 25 patients. Dave et al, ${ }^{[9]}$ in their cross sectional study conducted on 100 patients of diabetes mellitus, the fasting blood sugar (FBS) levels, HbA1C levels and serum lipid levels were performed and then correlated fasting blood sugar level with HbA1C levels and FBS and HbA1C with serum lipid levels. This study demonstrated that HbA1C level was increased in diabetics and it showed correlation with the status of control of diabetes. Diabetics have got increased level of serum cholesterol, triglycerides and decreased levels of serum HDL-cholesterol. HbA1C showed stronger correlation with serum cholesterol and triglycerides as compared to FBS. HDL-cholesterol showed 
stronger correlation with FBS than HbA1C.

We found that mean fasting blood glucose level in patients with good degree of control was 106.2, in fair was 164.4 and in poor was 208.2. Glycosylated hemoglobin level was good (6.74), fair (8.12) and poor (12.54). A positive correlation of fasting blood glucose and glycosylated hemoglobin level $(\mathrm{P}<$ 0.05) was found. Compagnucci et al, ${ }^{[10]}$ observed that during periods of wide fluctuations in blood sugar, HbA1C level remains nearly constant and in these patients measurement of $\mathrm{HbA} 1 \mathrm{C}$ is much more valuable in providing the assessment of diabetic control not available from random blood sugar measurements.

\section{Conclusion}

Authors found positive correlation of fasting blood glucose level and glycosylated hemoglobin level in patients with type II diabetes mellitus patients.

\section{References}

1. Reimer $\mathrm{F}$ et al: Incidence of hyperlipoproteinemia in patients with chemical and clinical diabetes. KlinWochenshr 1973; 51:973.

2. Gabbay K.H., Hasty K., Breslow J.L., Ellison R.C., Bunn H.F. and
Gallop P.M. Glycosylated haemoglobin and long term blood glucose control in diabetes mellitus. J. Clin.Endocr. Meta 1977;44:859.

3. Ricketts HT. Derangement vascular disease in diabetes Am J Med 1955;29:933.

4. Sharma D, Bansal BC, Prakash C. Serum lipid studies in insulin dependent diabetes below the age of 30 years. JIMA 1970;54:416.

5. Sosenko JM, Breslow JL and Miettinen OS. Hyperglycemia and plasma lipid levels. A prospective study of insulin dependent diabetic patients. New Eng J Med 1980; 302:650.

6. Pietri A, Dunn FL and Raskin P. The effect of improved diabetic control on plasma lipid and lipoprotein level: a comparison of conventional therapy and continuous subcutaneous insulin infusion. Diabetes 1980; 29:1001- 5 .

7. Somogyi M. and Nelson. Determination of blood sugar. J. Biochem. 19: 160, 1945.

8. Paulsen E.P. Glycosylated haemoglobin in childhood diabetes. Metabolism 1973;22: 269.

9. Mahesh Dave, Ajay Kumar Gupta, Puneet Patel, Heernath. Correlation between fasting blood sugar level, HbA1C level and serum lipid levels in type 2 diabetes mellitus patients. International Journal of Contemporary Medical Research 2019;6(7):26-29.

10. Compagnucci P., Cartechni M.G., Bolli G. et al. The importance of determining irreversible glycosylated haemoglobin in diabetes. Diabetes 1981;30:607. 14.

11. Elkeles R.S., Wu J. and Hambley J. Glycosylated haemoglobin, blood glucose and HDL-cholesterol in insulin requiring diabetics. Lancet 1978:2: 547.

12. Nabarro J.D.N., Mustaffa B.E., Morris D. et al. Insulin deficient diabetes. Diabetologia 1979;16:5.

Copyright: (C) the author(s), 2019. It is an open-access article distributed under the terms of the Creative Commons Attribution License (CC BY 4.0), which permits authors to retain ownership of the copyright for their content, and allow anyone to download, reuse, reprint, modify, distribute and/or copy the content as long as the original authors and source are cited.

How to cite this article: Chauhan NS, Agrawal HK. To Assess Correlation of Fasting Blood Glucose, Hb1 Ac and Serum Lipid Level in Type II Diabetes Mellitus Patients. Acad. J.Med.2019; 2(2):8-10.

DOI: dx.doi.org/10.21276/ajm.2019.2.2.3

Source of Support: Nil, Conflict of Interest: None declared. 